\title{
Suicidal Ideation Predicts Functioning and Quality of Life Over One Year after Acute Coronary Syndrome
}

\author{
Jae-Min Kim¹, Ju-Wan Kim¹, Hee-Ju Kang1', Kyung-Yeol Bae', Sung-Wan Kim¹, II-Seon Shin', \\ Young Joon Hong ${ }^{2}$, Youngkeun Ahn ${ }^{2}$, Myung Ho Jeong ${ }^{2}$, and Jin-Sang Yoon ${ }^{1} \bowtie$ \\ 1'Department of Psychiatry, Chonnam National University Medical School, Gwangju, Republic of Korea \\ ${ }^{2}$ Department of Cardiology, Chonnam National University Medical School, Gwangju, Republic of Korea
}

Objective This study investigated the associations of suicidal ideation (SI) evaluated within 2 weeks after an acute coronary syndrome (ACS) episode with functioning, disability, and quality of life (QOL) at a 1-year follow-up assessment.

Methods This study recruited 1152 consecutive patients within 2 weeks of a confirmed ACS episode; 828 of these patients who were followed up 1 year later comprised the study sample. SI was determined at baseline using the "suicidal thoughts" item of the MontgomeryÅsberg Depression Rating Scale. At both examinations, social and occupational functioning were measured by the Social and Occupational Functioning Assessment Scale (SOFAS), disability was estimated by World Health Organization Disability Assessment Schedule-12 (WHODAS-12), and QOL was assessed using the World Health Organization Quality of Life-Abbreviated form (WHOQOL-BREF). Baseline covariates included sociodemographic data, depression characteristics, cardiovascular risk factors, and current cardiac status.

Results SI at baseline was independently associated with less improved or decreased scores on the SOFAS, WHODAS-12, and WHOQOL-BREF over 1 year after adjusting for relevant covariates.

Conclusion SI within 2 weeks of an ACS episode predicted poorer functioning and QOL at a 1-year follow-up assessment. Thus, the simple evaluation of SI in patients with recently developed ACS could be helpful in screening for functioning and QOL during the chronic phase of this disease.

Psychiatry Investig 2019;16(1):65-70

Key Words Acute coronary syndrome, Disability, Quality of life, Social adjustment, Suicidality.

\section{INTRODUCTION}

Suicide is a major public health problem worldwide, ${ }^{1}$ and suicidal behavior has been associated with poor mental health. ${ }^{2}$ In particular, suicidal behavior is strongly associated with depression, in that such behavior strongly predicted depression relapse and persistence in depressive patients. ${ }^{34}$ Suicidal behavior may also be associated with functional aspects of mental health such as social functioning, disability, and quality of life (QOL). However, this issue has rarely been investigated, and most studies have been cross-sectional in design. ${ }^{5,6}$

Received: September 26, 2018 Accepted: October 22, 2018

$\triangle$ Correspondence: Jin-Sang Yoon, MD, PhD

Department of Psychiatry, Chonnam National University Medical School, 77 Yongbong-ro, Buk-gu, Gwangju 61186, Republic of Korea

Tel: +82-62-220-6142, Fax: +82-62-225-2351

E-mail: jsyoon@chonnam.ac.kr

(a) This is an Open Access article distributed under the terms of the Creative Commons Attribution Non-Commercial License (https://creativecommons.org/licenses/bync/4.0) which permits unrestricted non-commercial use, distribution, and reproduction in any medium, provided the original work is properly cited.
Suicidal behavior occurs more frequently in patients with severe physical disorders than in the general population. ${ }^{7}$ In particular, epidemiological and clinical studies have demonstrated that patients with acute coronary syndrome (ACS) are at increased risk for suicidal behavior. ${ }^{89}$ Therefore, the association between suicidal behavior and worse mental health may be more prominent in patients with ACS. In this regard, we previously reported that suicidal ideation (SI) within 2 weeks after an ACS episode predicted poorer outcomes on measures of depression at a 1-year follow-up assessment, similar to findings for patients with depression. ${ }^{10}$ However, no studies have investigated the effects of suicidal behavior on the functional aspects of mental health in patients with ACS, although treatment becomes focused on improving general health and functional status after the acute stage of ACS. ${ }^{11}$

The spectrum of suicidal behavior ranges from SI and attempts to completion. ${ }^{12}$ In clinical studies of ACS, SI was typically used as a phenotype. ${ }^{9,13}$ Thus, the present study investigated the effects of SI, identified within 2 weeks of an ACS episode, on social functioning, disability, and QOL at a 1-year 
follow-up assessment.

\section{METHODS}

\section{Study outline and participants}

The present analysis was carried out using data from the Korean DEPression in ACS (K-DEPACS) study, which investigated the epidemiology of depressive disorders in ACS patients using a naturalistic prospective design. The study design and rationale have been published. ${ }^{14,15}$ Of 4809 patients in the Department of Cardiology of Chonnam National University Hospital (CNUH) in Gwangju, South Korea, from 2006 to 2013 with recently developed ACS, 1152 met the eligibility criteria and agreed to participate in the study. Baseline evaluations made within 2 weeks after hospitalization for ACS included SI, sociodemographic and clinical characteristics, and functional status in terms of social functioning, disability, and QOL. At 1 year after the baseline evaluation, 828 patients were successfully followed up; this group comprised the present study sample. Follow-up evaluations were conducted in an outpatient setting, and the same functional status variables were assessed. Written informed consent was collected from each participant, and the study was approved by the CNUH Institutional Review Board (IRB No. 06-050).

\section{Suicidal ideation}

The presence of SI was identified using the "suicidal thoughts" item of the Montgomery-Åsberg Depression Rating Scale (MADRS-ST). ${ }^{16}$ This item assesses the feeling that life is not worth living and the existence of plans for suicide, with scores ranging from 0 (life satisfaction) to 6 (explicit plans for suicide). As in previous studies, ${ }^{17,18}$ we considered suicidal thoughts as being present if the patient scored $\geq 2$ on this item, where a score of 2 refers to fleeting suicidal thoughts.

\section{Sociodemographic and clinical characteristics}

Sociodemographic data on age, gender, duration of education, living status (living alone or not), housing (owned or rented), and current occupation (employed or not) were obtained. With regard to depression characteristics, previous and family histories of depression and scores for depressive disorder based on the Diagnostic and Statistical Manual (DSM)-IV were collected. ${ }^{19}$ The following cardiovascular risk factors were evaluated: diagnosed hypertension and diabetes mellitus, hypercholesterolemia by fasting serum total cholesterol level (>200 mg/dL), obesity by measured body mass in$\operatorname{dex}$ (BMI) $\left(>25 \mathrm{~kg} / \mathrm{m}^{2}\right)$, reported current smoking status, and previous and family histories of ACS. As a measure of current cardiac status, the severity of ACS was estimated by the Killip classification, ${ }^{20}$ left ventricular ejection fraction (LVEF) was estimated using echocardiography, and serum cardiac biomarkers troponin I and creatine kinase-MB (CK-MB) were measured.

\section{Functional status}

Social and occupational functioning was measured by the Social and Occupational Functioning Assessment Scale (SOFAS), ${ }^{21}$ which takes into account functional impairment caused by both physical limitations and mental problems. The SOFAS considers social and occupational functioning across a continuum that ranges from 100 (optimal functioning) to 1 (serious functional impairment). Disability was estimated using the World Health Organization Disability Assessment Schedule-12 (WHODAS-12). ${ }^{22}$ The WHODAS- 12 covers six domains: cognition, mobility, self-care, getting along, life activities, and participation. For each of the 12 items of WHODAS-12, respondents must rate their ability to undertake the addressed activities during the previous 30 days using a 5-point scale (no difficulty-extreme difficulty). A summary index is available, with scores ranging from 0 to 100; higher scores indicate higher disability. QOL was assessed by the World Health Organization Quality of Life-Abbreviated form (WHOQOLBREF), a 26-item self-administered questionnaire with items rated on a 5-point scale. ${ }^{23}$ WHOQOL-BREF evaluates four domains: physical factors, psychological factors, social relationships, and environmental context. The physical domain includes seven items including pain and discomfort; the psychological domain includes six items including positive affect; the social domain includes three items including personal relationships; and the environmental domain includes eight items including financial resources and transportation. Because the psychological domain includes depressive symptoms, this subscale was excluded, and the remaining three subscales were analyzed. Raw subscale scores were converted to a scale ranging from 0 to 100 to facilitate comparison with other instruments; final scores represented the percentage of the total possible score achieved, with higher scores indicating better QOL.

\section{Statistical analyses}

Baseline sociodemographic data, depression characteristics, cardiovascular risk factors, and current cardiac status were compared between patients with and without SI using either t-tests or chi-squared $\left(\chi^{2}\right)$ tests. The associations of SI at baseline with changes in the scores on SOFAS, WHODAS-12, and WHOQOL-BREF (values at 1 year minus values within 2 weeks after ACS onset) were evaluated using repeated-measures analysis of covariance to calculate group $\times$ time interactions after adjusting for corresponding baseline scores and covariates associated with SI at baseline $(\mathrm{p}<0.05)$. The Bonferroni 
correction was used to maintain an overall type I error rate of 0.05 against multiple comparisons for four Leeds Sleep Evaluation Questionnaire (LSEQ) outcomes; a two-sided p-value of $0.01(0.05 / 5)$ was used to define statistical significance. Statistical analyses were carried out using SPSS 21.0 software (IBM Corp., Armonk, NY, USA).

\section{RESULTS}

\section{Socio-economic and clinical characteristics by suicidal ideation}

SI at baseline was present in 164 (19.8\%) of the 828 study participants. Table 1 presents baseline characteristics of those

Table 1. Baseline characteristics by suicidal ideation $(\mathrm{SI})$ in 828 patients with acute coronary syndrome (ACS)

\begin{tabular}{|c|c|c|c|c|}
\hline & Absent SI $(\mathrm{N}=664)$ & Present SI $(\mathrm{N}=164)$ & Statistical coefficient & p-value* \\
\hline \multicolumn{5}{|l|}{ Socio-demographic characteristics } \\
\hline Age, mean (SD) years & $57.7(11.1)$ & $59.4(10.6)$ & $\mathrm{t}=-1.815$ & 0.070 \\
\hline Gender, N (\%) female & $173(26.1)$ & $64(39.0)$ & $\chi^{2}=10.83$ & 0.001 \\
\hline Education, mean (SD) years & $10.1(4.6)$ & $8.8(4.4)$ & $\mathrm{t}=+3.300$ & 0.001 \\
\hline Living alone, $\mathrm{N}(\%)$ & $53(8.0)$ & $18(11.0)$ & $\chi^{2}=1.503$ & 0.220 \\
\hline Housing, $N(\%)$ rented & $96(14.5)$ & $41(25.0)$ & $\chi^{2}=10.59$ & 0.001 \\
\hline Currently unemployed, N (\%) & $230(34.6)$ & $77(47.0)$ & $\chi^{2}=8.546$ & 0.003 \\
\hline \multicolumn{5}{|l|}{ Depression characteristics, N (\%) } \\
\hline Previous depression & $23(3.5)$ & $15(9.1)$ & $\chi^{2}=9.699$ & 0.002 \\
\hline Family history of depression & $20(3.0)$ & $9(5.5)$ & $\chi^{2}=2.385$ & 0.122 \\
\hline DSM-IV depressive disorder & $191(28.8)$ & $133(81.1)$ & $\chi^{2}=151.2$ & $<0.001$ \\
\hline \multicolumn{5}{|l|}{ Cardiac risk factors, $\mathrm{N}(\%)$} \\
\hline Hypertension & $305(45.9)$ & $81(49.4)$ & $\chi^{2}=0.631$ & 0.427 \\
\hline Diabetes mellitus & $129(19.4)$ & $39(23.8)$ & $\chi^{2}=1.541$ & 0.215 \\
\hline Hypercholesterolemia & $349(52.6)$ & $92(56.1)$ & $\chi^{2}=0.661$ & 0.416 \\
\hline Obesity & $301(45.3)$ & $63(38.4)$ & $\chi^{2}=2.554$ & 0.110 \\
\hline Current smoker & $255(38.4)$ & $56(34.1)$ & $\chi^{2}=1.016$ & 0.313 \\
\hline Previous ACS & $28(4.2)$ & $7(4.3)$ & $\chi^{2}=0.001$ & 0.977 \\
\hline Family history of ACS & $19(2.9)$ & $8(4.9)$ & $\chi^{2}=1.695$ & 0.193 \\
\hline \multicolumn{5}{|l|}{ Current cardiac status } \\
\hline Killip class >1, N (\%) & $103(15.5)$ & $33(20.1)$ & $\chi^{2}=2.036$ & 0.154 \\
\hline LVEF, mean (SD) \% & $61.5(11.2)$ & $60.4(11.1)$ & $\mathrm{t}=+1.125$ & 0.261 \\
\hline Troponin I, mean (SD) mg/dL & $9.1(15.5)$ & $12.0(14.5)$ & $\mathrm{t}=-2.160$ & 0.036 \\
\hline CK-MB, mean (SD) mg/dL & $17.6(41.5)$ & $18.7(27.7)$ & $\mathrm{t}=-0.330$ & 0.742 \\
\hline
\end{tabular}

${ }^{*}$ p-values using t-tests or $\chi^{2}$ tests as appropriate. DSM-IV: Diagnositic and Statistical Manual of Mental Disorders, 4th edition, LVEF: left ventricular ejection fraction, CK-MB: creatine kinase-MB, SD: standard deviation

Table 2. Scale scores by baseline suicidal ideation $(\mathrm{SI})$ at baseline and at follow-up $(\mathrm{N}=828)$

\begin{tabular}{|c|c|c|c|c|c|c|}
\hline & \multicolumn{2}{|c|}{ Absent SI $(\mathrm{N}=664)$} & \multicolumn{2}{|c|}{ Present SI (N=164) } & \multicolumn{2}{|c|}{ Statistics for group $\times$ time interaction* } \\
\hline & At baseline & At follow-up & At baseline & At follow-up & $\mathrm{F}$ & $\mathrm{p}$ \\
\hline SOFAS & $79.0(6.8)$ & $80.7(7.9)$ & $71.6(9.2)$ & $69.5(9.1)$ & 19.15 & $<0.001^{\dagger}$ \\
\hline WHODAS-12 & $14.2(16.4)$ & $8.7(14.7)$ & $31.2(20.4)$ & $31.0(18.6)$ & 9.117 & $0.003^{\dagger}$ \\
\hline \multicolumn{7}{|l|}{ WHOQOL-BREF } \\
\hline Physical factors & $54.8(13.5)$ & $59.1(16.4)$ & $41.5(14.4)$ & $41.7(16.7)$ & 8.538 & $0.004^{\dagger}$ \\
\hline Social relationships & $60.0(12.6)$ & $62.1(14.1)$ & $48.5(16.3)$ & $47.2(17.7)$ & 5.841 & 0.016 \\
\hline Environmental context & $56.2(12.7)$ & $58.5(12.9)$ & $46.8(13.5)$ & $45.6(15.4)$ & 7.614 & $0.006^{\dagger}$ \\
\hline
\end{tabular}

Data are mean (SD) scores. *repeated measures analysis of covariance in a mixed model with corresponding baseline scores, gender, education, housing, current employment, past history of depression, DSM-IV depressive disorder, and troponin I as covariates, ${ }^{\dagger}$ statistical significance after applying Bonferroni correction. SOFAS: Social and Occupational Functioning Assessment Scale, WHODAS-12: World Health Organization Disability Assessment Schedule-12, WHOQOL-BREF: World Health Organization Quality of Life-Abbreviated form 
with and without SI. SI was significantly associated with female gender, lower education, rented housing, current unemployment, previous history of depression, DSM-IV depressive disorder, and higher troponin-I levels. Table 2 summarizes adjusted associations between simultaneously entered baseline characteristics and suicidal ideation. In the acute phase of ACS, suicidal ideation was independently associated with past history of depression, depressive and anxiety disorders, and higher troponin-I levels.

\section{Associations of SI at baseline with changes in functional status over 1 year}

The associations between SI within 2 weeks of the ACS episode and changes in functional status over 1 year are summarized in Table 2 and illustrated in Figure 1. SI at baseline was independently associated with less improvement or a decrease in the scores on SOFAS, WHODAS-12, and all three domains of WHOQOL-BREF at 1 year post-ACS after adjusting for gender, education, housing, current employment, previous history of depression, DSM-IV depressive disorder, and troponin I levels. After applying the Bonferroni correction, the associations remained significant for SOFAS, WHODAS-12, and the physical and environmental domains of WHOQOLBREF in the same adjusted model.

\section{DISCUSSION}

The principal findings of this study were that documented SI within 2 weeks after ACS independently predicted less improvement or a decrease in social functioning, increased disability, and reduced QOL during the 1-year follow-up period. These findings were robust after adjustment for a range of demographic and clinical covariates related to SI, including depression characteristics.

In the acute phase of ACS, there is pressing need for intensive medical treatment to save the patient's life and minimize sequelae. ${ }^{24}$ However in the chronic phase of ACS, priority is given to treatment aimed at improving functional status. ${ }^{11}$ Previous studies have found only minimal or no association between cardiac disease severity and functional status. ${ }^{25,26}$ Rather, depression has been identified as the most significant factor predicting functional status in ACS. ${ }^{27,28}$ Other psychosocial factors including life stress or social support also predicted functional status in ACS. ${ }^{29-31}$ The present study is the first to

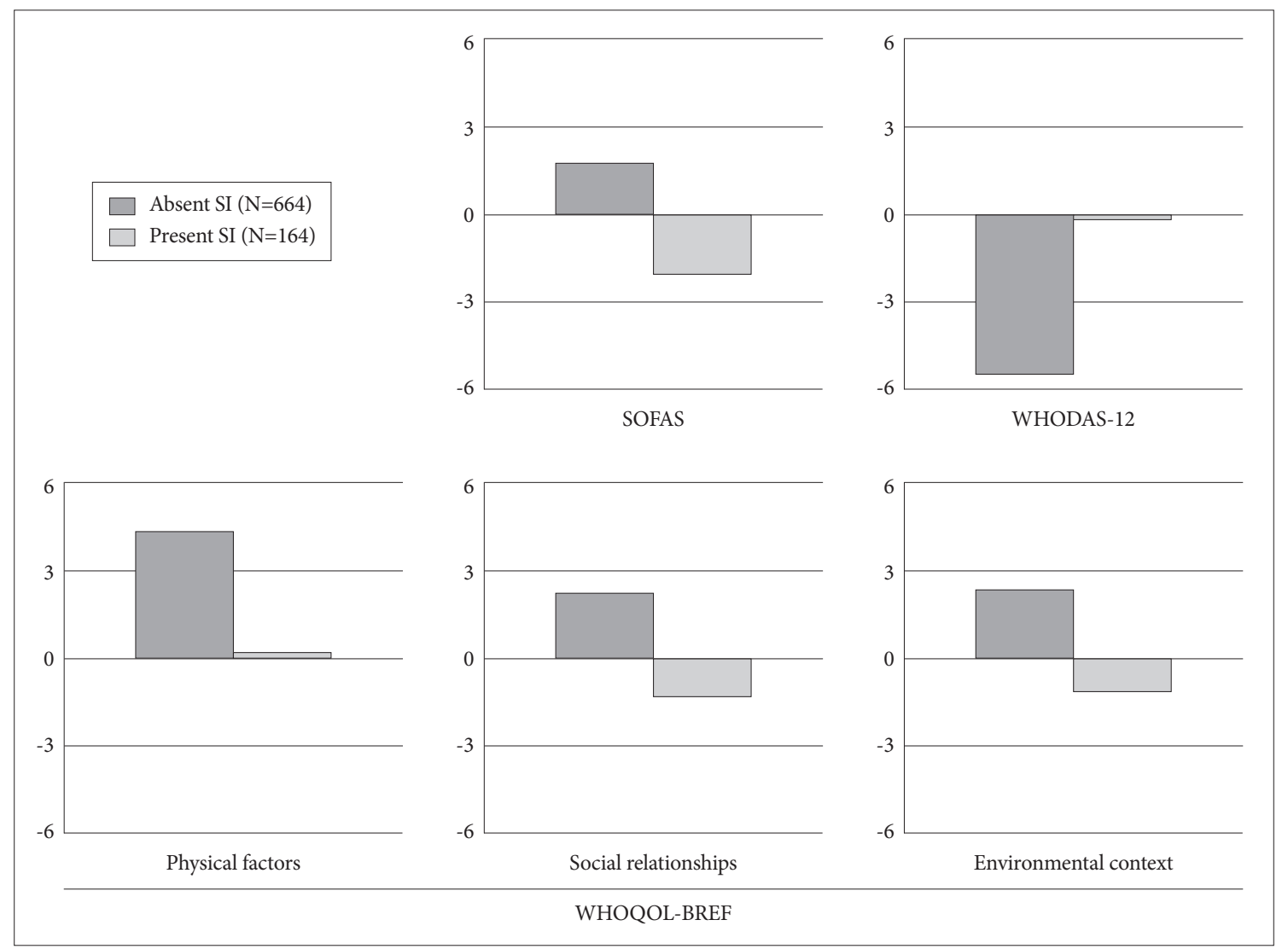

Figure 1. Changes in scale scores by baseline suicidal ideation ( $\mathrm{SI})$ over one year follow-up $(\mathrm{N}=828)$. SOFAS: Social and Occupational Functioning Assessment Scale, WHODAS-12: World Health Organization Disability Assessment Schedule-12, WHOQOL-BREF: World Health Organization Quality of Life-Abbreviated form. 
report the predictive value of SI for functional status in ACS. This finding might be expected due to the fact that SI is one of the diagnostic criteria for depressive disorders, ${ }^{19}$ so SI may be fully explained by depression. However, previous research has suggested that pathophysiological characteristics of SI are independent of underlying psychiatric disorders, including depression. ${ }^{32,33}$ Moreover, the associations of SI with elements of functional status remained significant even after adjustment for depression characteristics. Screening for depression is conventionally recommended for all patients with ACS because this disorder is significantly associated with increased psychiatric morbidity and mortality. ${ }^{34}$ The present findings suggest that screening for SI may also be important for ACS patients due to the predictive value of SI at baseline for functional status 1 year later.

Before drawing conclusions from the findings of the present study, several methodological issues should be considered. First, the main exposure variable was SI, and although SI is closely related to more severe suicidal behaviors, ${ }^{35}$ no data concerning suicide attempts were obtained. However, previous clinical studies of patients with ACS or other severe physical disorders have also used SI as a phenotype, as more severe forms of suicidal behavior are rare in this type of study. ${ }^{9,36}$ Second, SI was identified using the "suicidal thoughts" item of the MADRS rather than being defined using a separate or formal instrument. However, this method has been used in previous studies investigating patients with severe physical disorders as well as patients with depressive disorders. ${ }^{14,17,37}$ Furthermore, a recent study reported that an item pertaining to suicidal thoughts taken from a depression rating scale had good validity. ${ }^{38}$ Third, it is important to bear in mind that the follow-up assessment was performed at only a single time point and not throughout the 1-year period; thus, it was impossible to determine the nature or course of functional status between examinations.

This study has several strengths. First, participants were recruited consecutively from among all eligible patients with recent ACS, and all measurements were assessed at similar time points (i.e., at 2 weeks and 1 year after ACS), which reduced the risk of error arising from heterogeneous examination times. Second, all measurements of psychiatric and cardiovascular status were well validated. Third, functional outcome was assessed across three comprehensive concepts: social function, disability, and QOL. Fourth, a range of covariates was considered in the analyses.

ACS is the leading cause of disease burden worldwide. ${ }^{39}$ The present findings suggest that the simple evaluation of SI in patients with recently developed ACS in a hospital setting could be helpful in screening for functional status over 1 year of this disease. The long-term effects of SI on the treatment, course, and prognosis of ACS should be determined in future research.

\section{Acknowledgments}

This study was supported by grants of the Korea Health 21 R\&D, Ministry of Health and Welfare, Republic of Korea (HI12C0035), and was supported by Basic Science Research Program through the National Research Foundation of Korea (NRF) funded by the Ministry of Science, ICT and future Planning (NRF-2016R1A2A2A05919518).

\section{REFERENCES}

1. WHO. World Report on Violence and Health. Geneva: World Health Organization; 2012.

2. Mann J. A current perspective on suicide and attempted suicide. Ann Intern Med 2002;136:302-311

3. Blackmore ER, Munce S, Weller I, Zagorski B, Stansfeld SA, Stewart $\mathrm{DE}$, et al. Psychosocial and clinical correlates of suicidal acts: results from a national population survey. Br J Psychiatry 2008;192:279-284.

4. Oquendo MA, Currier D, Mann JJ. Prospective studies of suicidal behavior in major depressive and bipolar disorders: what is the evidence for predictive risk factors? Acta Psychiatr Scand 2006;114:151-158.

5. Canal-Rivero M, Lopez-Moriñigo JD, Barrigón ML, Ayesa-Arriola R, Crespo-Facorro B, Ruiz-Veguilla M, et al. Suicidal behavior and personality traits contribute to disability in first-episode psychosis: a 1-year follow-up study. Suicide Life Threat Behav 2018 [Epub ahead of print].

6. Oladeji BD, Taiwo B, Mosuro O, Fayemiwo SA, Abiona T, Fought AJ, et al. Suicidal behavior and associations with quality of life among HIV-infected patients in Ibadan, Nigeria. J Int Assoc Provid AIDS Care 2017;16:376-382.

7. Kang HJ, Kim SY, Bae KY, Kim SW, Shin IS, Yoon JS, et al. Comorbidity of depression with physical disorders: research and clinical implications. Chonnam Med J 2015;51:8-18.

8. Larsen KK, Agerbo E, Christensen B, Søndergaard J, Vestergaard M. Myocardial infarction and risk of suicide: a population-based case-control study. Circulation 2010;122:2388-2393.

9. Nascimento ER, Maia AC, Soares-Filho G, Nardi AE, Cardoso A. Predictors of suicidal ideation in coronary artery disease. Compr Psychiatry 2015;57:16-20.

10. Kim JM, Kang HJ, Bae KY, Kim SW, Shin IS, Yoon JS, et al. Association of suicidal ideation with depression 1 year after an acute coronary syndrome episode. Gen Hosp Psychiatry 2017;45:101-102.

11. Ruo B, Rumsfeld JS, Hlatky MA, Liu H, Browner WS, Whooley MA. Depressive symptoms and health-related quality of life: the Heart and Soul Study. J Am Med Assoc 2003;290:215-222.

12. Beck A, Kovacs M, Weissman M. Assessment of suicidal ideation: the scale for suicide ideation. J Consult Clin Psychol 1979;47:343-352.

13. Kishi Y, Robinson RG, Kosier JT. Suicidal ideation among patients with acute life-threatening physical illness: patients with stroke, traumatic brain injury, myocardial infarction, and spinal cord injury. Psychosomatics 2001;42:382-390.

14. Kim JM, Bae KY, Kang HJ, Kim SW, Shin IS, Hong YJ, et al. Design and methodology for the Korean observational and escitalopram treatment studies of depression in acute coronary syndrome: K-DEPACS and EsDEPACS. Psychiatry Investig 2014;11:89-94.

15. Kim JM, Stewart R, Lee YS, Lee HJ, Kim MC, Kim JW, et al. Effect of escitalopram vs placebo treatment for depression on long-term cardiac outcomes in patients with acute coronary syndrome: the DEPACS randomized clinical trial. JAMA 2018:320:350-358.

16. Montgomery SA, Asberg M. A new depression scale designed to be sensitive to change. Br J Psychiatry 1979;134:382-389.

17. Murrough JW, Soleimani L, DeWilde KE, Collins KA, Lapidus KA, Iacoviello BM, et al. Ketamine for rapid reduction of suicidal ideation: a 
randomized controlled trial. Psychol Med 2015;45:3571-3580.

18. Santos CO, Caeiro L, Ferro JM, Fiqueira ML. A study of suicidal thoughts in acute stroke patients. J Stroke Cerebrovasc Dis 2012;21:749-754.

19. American Psychiatric Association. Diagnostic and Statistical Manual of Mental Disorders. 4th Ed. Washington, DC: American Psychiatric Press Inc; 1994.

20. Killip T 3rd, Kimball JT. Treatment of myocardial infarction in a coronary care unit. A two year experience with 250 patients. Am J Cardiol 1967;20:457-464.

21. Goldman HH, Skodol AE, Lave TR. Revising Axis V for DSM-IV: a review of measures of social functioning. Am J Psychiatry 1992;149: 1148-1156.

22. Eipping-Jordan J, Ustun TB. Assessment Classification and epidemiology Group. The WHODAS II: levelling the playing field for all disorders. WHO Ment Health Bull 2000;6:5-6.

23. Development of the World Health Organization WHOQOL-BREF quality of life assessment. The WHOQOL Group. Psychol Med 1998;28:551558.

24. Anderson JL, Adams CD, Antman EM, Bridges CR, Califf RM, Casey DE Jr, et al. 2012 ACCF/AHA focused update incorporated into the ACCF/AHA 2007 guidelines for the management of patients with unstable angina/non-ST-elevation myocardial infarction: a report of the American College of Cardiology Foundation/American Heart Association Task Force on Practice Guidelines. J Am Coll Cardiol 2013;61: e179-e347.

25. Rumsfeld JS, Havranek E, Masoudi FA, Peterson ED, Jones P, Tooley JF, et al. Depressive symptoms are the strongest predictors of shortterm declines in health status in patients with heart failure. J Am Coll Cardiol 2003;42:1811-1817.

26. Sin NL, Yaffe K, Whooley MA. Depressive symptoms, cardiovascular disease severity, and functional status in older adults with coronary heart disease: the heart and soul study. J Am Geriatr Soc 2015;63:8-15.

27. Dickens CM, McGowan L, Percival C, Tomenson B, Cotter L, Heagerty $\mathrm{A}$, et al. Contribution of depression and anxiety to impaired healthrelated quality of life following first myocardial infarction. Br J Psychiatry 2006;189:367-372.

28. Kim JM, Stewart R, Bae KY, Kang HJ, Kim SW, Shin IS, et al. Effects of depression co-morbidity and treatment on quality of life in patients with acute coronary syndrome: the Korean depression in ACS (K-DEPACS) and the escitalopram for depression in ACS (EsDEPACS) study. Psychol
Med 2015;45:1641-1652.

29. Albus C. Psychological and social factors in coronary heart disease. Ann Med 2010;42:487-494.

30. Arnold SV, Smolderen KG, Buchanan DM, Li Y, Spertus JA. Perceived stress in myocardial infarction: long-term mortality and health status outcomes. J Am Coll Cardiol 2012;60:1756-1763.

31. Bucholz EM, Rathore SS, Gosch K, Schoenfeld A, Jones PG, Buchanan $\mathrm{DM}$, et al. Effect of living alone on patient outcomes after hospitalization for acute myocardial infarction. Am J Cardiol 2011;108:943-948.

32. Powell J, Geddes J, Deeks J, Goldacre M, Hawton K. Suicide in psychiatric hospital in-patients: risk factors and their predictive power. $\mathrm{Br} \mathrm{J}$ Psychiatry 2000;176:266-272.

33. Fu Q, Heath AC, Bucholz KK, Nelson EC, Glowinski AL, Goldberg J, et al. A twin study of genetic and environmental influences on suicidality in men. Psychol Med 2002;32:11-24.

34. Lichtman JH, Bigger JT Jr, Blumenthal JA, Frasure-Smith N, Kaufmann PG, Lespérance F, et al. Depression and coronary heart disease: recommendations for screening, referral, and treatment: a science advisory from the American Heart Association Prevention Committee of the Council on Cardiovascular Nursing, Council on Clinical Cardiology, Council on Epidemiology and Prevention, and Interdisciplinary Council on Quality of Care and Outcomes Research: endorsed by the American Psychiatric Association. Circulation 2008;118:1768-1775.

35. Posner K, Brown GK, Stanley B, Brent DA, Yershova KV, Oquendo MA, et al. The Columbia-Suicide Severity Rating Scale: Initial validity and internal consistency finding $s$ from three multisite studies with adolescents and adults. Am J Psychiatry 2011;168:266-277.

36. Kim JM, Kim SW, Kang HJ, Bae KY, Shin IS, Kim JT, et al. Serotonergic genes and suicidal ideation 2 weeks and 1 year after stroke in Korea. Am J Geriatr Psychiatry 2014;22:980-988.

37. Kim JM, Jang JE, Stewart R, Kim SY, Kim SW, Kang HJ, et al. Determinants of suicidal ideation in patients with breast cancer. Psychooncology 2013;22:2848-2856.

38. Green KL, Brown GK, Jager-Hyman S, Cha J, Steer RA, Beck AT. The predictive validity of the beck depression inventory suicide item. J Clin Psychiatry 2015;76:1683-1686.

39. Murray CJ, Vos T, Lozano R, Naghavi M, Flaxman AD, Michaud C, et al. Disability-adjusted life years (DALYs) for 291 diseases and injuries in 21 regions, 1990-2010: a systematic analysis for the Global Burden of Disease Study 2010. Lancet 2012;380:2197-2223. 